\title{
The Analysis of Thermomechanical Periodic Motions of a Drinking Bird
}

\author{
Schun T. Uechi ${ }^{1}$, Hiroshi Uechi ${ }^{2}$, Akihiko Nishimura ${ }^{3}$ \\ ${ }^{1}$ KPMG Ignition Tokyo, Data Tech., Tokyo, Japan \\ ${ }^{2}$ Osaka Gakuin University, Suita, Osaka, Japan \\ ${ }^{3}$ Japan Atomic Energy Agency, Tokai-mura, Ibaraki, Japan \\ Email: quantum2006hara@gmail.com, uechi@ogu.ac.jp,nishimura.akihiko@jaea.go.jp
}

How to cite this paper: Uechi, S.T., Uechi, H. and Nishimura, A. (2019) The Analysis of Thermomechanical Periodic Motions of a Drinking Bird. World Journal of Engineering and Technology, 7, 559-571. https://doi.org/10.4236/wjet.2019.74040

Received: August 12, 2019

Accepted: September 22, 2019

Published: September 25, 2019

Copyright (c) 2019 by author(s) and Scientific Research Publishing Inc. This work is licensed under the Creative Commons Attribution International License (CC BY 4.0).

http://creativecommons.org/licenses/by/4.0/ cc) (i) Open Access

\begin{abstract}
A water drinking bird or simply drinking bird (DB) is discussed in terms of a thermomechanical model. A mathematical expression of motion derived from the thermomechanical model of a drinking bird and numerical solutions are explicitly shown, which is helpful in understanding physical meanings and fundamental difference between mechanical and thermomechanical periodic motion. The mathematical and physical differences between mechanical and thermomechanical motions are clearly examined, resulting in time-independent and time-dependent coupling constants of equations of motion and continuous transitions between bifurcation solutions. The thermodynamical and irreversible process of a drinking bird motion could be theoretically examined and practically applied to energy harvesting technologies by way of the current modeling. As an example of irreversible thermodynamics, the thermomechanical model of DB will help understand heat engines manifested from microscopic to macroscopic systems.
\end{abstract}

\section{Keywords}

Mechanical and Thermomechanical Model, Nonlinear Equations, Continuous Transitions between Bifurcation Solutions, Energy Harvesting Technologies

\section{Introduction}

A drinking bird (DB) known as dunking bird, insatiable birdie or dipping bird is an interesting toy, which simultaneously demonstrates wonders of thermomechanical phenomena. It is reported that a physicist, A. Einstein, was so fascinated by the drinking bird that he spent for three and one-half months to figure 
out or enjoy the relation between evaporation-pressure and mechanical work [1]. The DB's motion displays a fundamental thermomechanical phenomenon, which is a fundamental and active research field at present.

The DB toy consists of the upper bulb (head), body tube and lower bulb filled with a highly volatile liquid of dichloromethane $\left(\mathrm{CH}_{2} \mathrm{Cl}_{2}\right)$ shown in Figure 1(a). The mechanical motion of a DB is given by a nonlinear differential equation coupled to a stream of thermal energy which assists the up-and-down motion of the volatile liquid substance in a glass tube, which is driven by the pressure difference exerted by temperature difference between head and lower bulbs. The temperature difference produced by evaporation of water around head and beak is essential to generate periodic motions as a whole, and so, the water drinking bird is actually regarded as a small heat engine.

Though a drinking bird is a heat engine, the motion has not been investigated through thermodynamics and theory of irreversible thermodynamics, and in addition, it is not clearly discussed which movements of a DB are respectively mechanical and thermodynamical. One reason can be stated that a physical modeling of a drinking bird has not been clearly shown, and only experimental measurements of motion have been conducted by using modern equipments, such as voltmeter, thermistor, magnetometer and a light shutter of a photovoltaic cell [2] [3] [4]. We discussed possible theoretical modeling of DB's motion for the theory of irreversible thermodynamics, which is a purpose of the current paper.

A Stirling engine is also a well-known heat engine operated by a cyclic compression and expansion of air at different temperatures, which could be an active research area in spaceship project for energy resources by using nuclear energy. In terms of thermodynamic principles, the drinking bird, Stirling engine and nuclear radiation reactors are considered to be equivalent as "heat engines" [5] [6] [7], and hence, one can learn fundamental principles of thermal reactors, or even applications to nuclear reactors from dynamics of a drinking bird. The flow of heat into environment or dissipation of heat into thermal bath is producing mechanical work, whose driving force is called the principle of maximum entropy in thermodynamics known as the second law of thermodynamics. Hence, it exhibits fundamental properties for physics; the water drinking bird is a marvelous toy not only for children but also for experts and people in general.

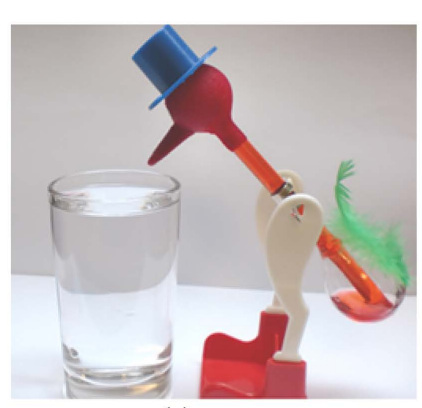

(a)

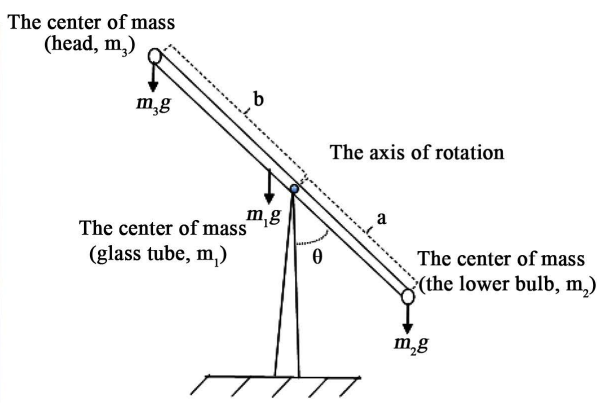

(b)

Figure 1. A mechanical modeling of a drinking bird. (a) A drinking bird (DB); (b) A mechanical model for the drinking bird of (a). 
Macroscopic motions and changes in nature including living things are considered to be consequences of nonlinear dynamical interactions based on energy and entropy [8] [9] [10], dissipative processes and streams of heat existing from macroscopic and thermomechanical structures to microscopic, cellular, molecular and atomic structures. The motion of a drinking bird is produced by nonlinear interactions which are difficult to trace from the solution of equations of motion. This is because a nonlinear differential equation usually produces bifurcation solutions which are qualitatively and quantitatively different from each other, which was first introduced by mathematician, Henri Poincaré, in 1885 [11]. The bifurcation solutions appear suddenly with changes of parameter values of a nonlinear equation, and even when a small smooth change is made to parameter values, one will observe a stable solution suddenly splitting into two, or possibly more stable solutions [12] [13] of nonlinear equations of classical and quantum systems.

The DB's mechanical nonlinear equation of motion has bifurcation solutions, for example, an ordinary periodic oscillation around $\theta=0$ (see, Figure 1), and an upside-down oscillation converging to $\theta=\pi$ (see, in Section 2), which is impossible to construct continuous solutions to connect bifurcation solutions because they are converging to different values. It is not possible for a mechanical DB model to obtain a periodic solution starting from an oscillation around $\theta=0$, then converging to $\theta=\pi$ by changing parameter values of the equation of motion (see, Section 2).

We introduced a thermomechanical model of $\mathrm{DB}$, which continuously connects bifurcation solutions and imitates DB's drinking motion reasonably well; the thermomechanical model clearly exhibits oscillations of lovable drinking bird. We introduce a thermomechanical model of water drinking bird by assuming a constant volatile-liquid velocity, $v_{0}$, in order to imitate DB's approximate periodic motions. The parameter values of the DB's nonlinear differential equation of motion are examined in the numerical analysis and solutions are explicitly exhibited. A mechanical model and numerical calculations are explained in Section 2, and a thermomechanical model with thermal driving force and numerical simulations are discussed in Section 3, which clearly shows that DB's motion is induced by Newtonian mechanics and irreversible thermodynamics. Conclusions and perspectives are discussed in Section 4.

\section{The Mechanical Motion of a Drinking Bird}

The DB's drinking period (time from a drinking to next drinking) changes drastically with environmental and mechanical conditions (temperature, humidity, friction of mechanical parts, impacts on the edge of glass, amount of water on the beak, ...). Hence, the average period of drinking bird can only be determined roughly. The periods in the beginning are rather long, which may take about 2 minutes with the initial starting angle $\theta=\pi / 6$ (see, 1(b)), and it becomes roughly converging to 30 - 40 seconds when oscillations are stable, depending on environmental and thermal conditions. All the periods of a drinking bird are not 
the same as those determined in mechanical pendulums and springs.

The periodic motion of a drinking bird is considered to be composed of two independent motions: a simple back and forth oscillation around $\theta=0$ and a drinking motion converging to $\theta=\pi$ which is an upside-down oscillation. However, the upside-down oscillation of a drinking bird is mechanically restricted in a real toy, not to go beyond an angle $\theta \sim \pi / 2$ by the shape of arm and a hole of axis of rotation (see Figure 1(a)). A simplified mechanical drinking bird is introduced as Figure 1(b) by employing the concept of centers of mass, and centers of mass of head, glass tube and lower bulb are respectively introduced as $m_{3}, m_{1}$ and $m_{2}\left(m_{1}+m_{2}+m_{3}=28.0 \mathrm{~g}\right)$. The length of mechanical drinking bird is $a+b=l$ and supposed to be $a \simeq 6.0, l \simeq 12.4(\mathrm{~cm})$ for numerical calculations. The cgs-unit ( $\mathrm{cm}$, gram, second) is used in the paper. This is the basic mechanical structure to be improved by a thermomechanical model for realistic simulations in Section 3.

The equation of motion for the mechanical drinking bird is constructed from Newtonian mechanics, or Lagrangian dynamics with the rotational kinetic energy and potential energy:

$$
\mathcal{L}=\frac{1}{2} I_{0} \dot{\theta}^{2}+\left\{m_{2} g a-m_{1} g\left(b-\frac{l}{2}\right)-m_{3} g b\right\} \cos \theta .
$$

where the moment of inertia is given by the sum, $I_{0}=I_{1}+I_{2}+I_{3}$, which consists of the moment of inertia of head $I_{3}$, glass tube $I_{1}$, and lower bulb $I_{2}$, respectively. Because of the center of mass assumption, the moment of inertia of head and lower bulb are given by $I_{2}=m_{2} a^{2}$ and $I_{3}=m_{3} b^{2}$. One should note that the mass, $m_{2}$, is the sum of masses of liquid and lower glass bulb.

The glass tube is assumed to have the mass $m_{1}$ with length $l=a+b$, and the moment of inertia is obtained as [14] [15]:

$$
I_{1}=m_{1} I^{2}\left(1 / 3-\left(1-\frac{b}{l}\right)+\left(1-\frac{b}{l}\right)^{2}\right) .
$$

The angle $\theta$ is measured from the vertical direction as in Figure 1(b), and the equation of motion for $\mathrm{DB}$ is derived by way of the Euler-Lagrange equation as,

$$
I_{0} \ddot{\theta}+g l\left\{m_{2} \frac{a}{l}-m_{1}\left(\frac{b}{l}-\frac{1}{2}\right)-m_{3} \frac{b}{l}\right\} \sin \theta=0,
$$

where $I_{0}=I_{1}+m_{2} a^{2}+m_{3} b^{2}$.

The velocity-dependent friction term, $c \dot{\theta}$ ( $c$ is a free parameter), is included in the equation of motion for accounting an oscillation damping, and by defining the effective mass, $m^{*}$, for convenience as:

$$
m^{*}=m_{2} \frac{a}{l}-m_{1}\left(\frac{b}{l}-\frac{1}{2}\right)-m_{3} \frac{b}{l},
$$

the mechanical equation of motion for a DB is written as,

$$
\ddot{\theta}+c \dot{\theta}+\frac{g l m^{*}}{I_{0}} \sin \theta=0 .
$$


Now, one can check the nonlinear Equation (2.5) whether it can express the motion of DB reasonably well or not by adjusting parameters, a,l,c, $m_{1}, m_{2}, m_{3}$; initial conditions, $\theta(0)=\pi / 6$ and $\dot{\theta}(0)=0$, are used in numerical simulations. The following parameters are fixed: $g=980\left(\mathrm{~cm} / \mathrm{s}^{2}\right), \quad a=6.0(\mathrm{~cm})$, $l=13.0(\mathrm{~cm}), \quad c=0.04(1 / \mathrm{s})$.

In the mechanical DB model, one obtains bifurcation solutions: a simple back and forth damping oscillation converging to $\theta=0$ and an upside-down damping oscillation converging to $\theta=\pi$, as shown in Figure 2(a) and Figure 2(b) respectively. The solutions are independent and suddenly change to the other solution when combinations of mass parameter values get to a threshold for a bifurcation. Typical solutions to the DB nonlinear mechanical equation are shown in Figure 2; $m_{1}=1.40, m_{2}=14.0, m_{3}=0.9 m_{2}$ for Figure 2(a), and $m_{1}=2.80, m_{2}=14.0, m_{3}=0.8 m_{2}$ for Figure $2(\mathrm{~b})$. The solution of upside-down motion is mechanically restricted in a DB toy as one can notice from the shape of arm and axis of rotation [16]. However, it is not restricted in the mechanical model of a DB, and hence, upside-down oscillation solutions (oscillations converging to $\theta=\pi$ or $\theta=-\pi$ ) can suddenly appear in certain combinations of parameter values, and it is not possible to find a continuous solution from Figure 2(a) to Figure 2(b) by changing parameters of the Equation (2.5), because they are mutually bifurcation solutions.

Based on the mechanical DB simulations, we remark:

(Remark 1)

The imitation and construction of the motion of drinking bird cannot be possible based only on Newtonian mechanics, because the nonlinear equations derived from Newtonian mechanics cannot describe continuous transition between bifurcation solutions.

The shape of a mechanical DB could be more realistic, which corresponds to changing distributions of mass and the moment of inertia. Changing parameters do not produce DB's periodic motion. Hence, different external force to keep
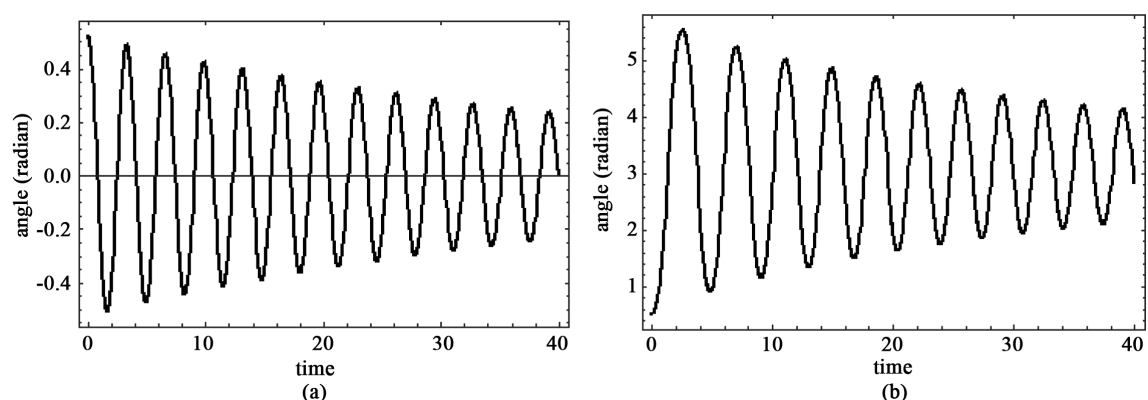

Figure 2. Solutions to a mechanical drinking bird defined by the Equation (5). (a) [The back and forth damping oscillation of a mechanical drinking bird. Initial values: $\theta(0)=\pi / 6, \dot{\theta}(0)=0, \quad$ (oscillations converging to $\theta=0$ ).]; (b) [The upside-down oscillation of a mechanical drinking bird. Initial values: $\theta(0)=\pi / 6, \dot{\theta}(0)=0$. (oscillations converging to $\theta=\pi$ )]. 
periodic motion should be essential, which originates from thermodynamic force. The properties of nonlinearity of DB equation make analyses of solutions difficult when thermal effects are included, but it results in a physically and mathematically interesting solution, which will be discussed and numerically shown in the following sections.

\section{A Thermomechanical Modeling for the Motion of a Drinking Bird}

We will demonstrate a method to imitate DB's periodic motion, which is beyond classical Newtonian mechanics as discussed in the previous section. From experimental observation, one can examine that physical parameters of equation are changing with time, because the liquid inside a glass tube $\left(\mathrm{CH}_{2} \mathrm{Cl}_{2}\right)$ is moving upward with time until the drinking bird drinks water. The DB's mechanical constituents which should become time-dependent would be, respectively, the moment of inertia, $I_{1}, I_{2}, I_{3}$, masses, $m_{1}, m_{2}, m_{3}$, and the center of gravity of $m_{2}$ fixed at $a$; the center of gravity of $m_{2}$ shifts upward because liquid in $m_{2}$ moves upward with time.

The constituents become time-dependent by way of upward moving liquid induced by external thermodynamic force. The velocity of upward moving liquid is not smooth, however, we assume the average velocity of liquid as $v_{0}$, whose appropriate value will be determined by numerical simulations. The mass and moment of inertia, $m_{1}=7.0(\mathrm{~g})$ and $I_{1}$ given by the Equation (2.2), are fixed with length $l=12.4(\mathrm{~cm})$ for simulations. The DB's motion is not sensitive to variations of mass $m_{1}$.

\subsection{The Construction of Time-Dependent Moment of Inertia, $I_{2}(t)$, Induced by Upward Moving Liquid}

Let us suppose that at time $t$, the volatile liquid (density, $\rho_{l}, \mathrm{~g} / \mathrm{cm}^{3}$ ) in the glass tube (cross-section, $s, \mathrm{~cm}^{2}$ ) goes upward with the speed $v_{0}$ and length, $v_{0} t$, as in Figure 3. The mass $m_{2}$ is separated to the upward moving liquid and the rest of liquid with the lower glass bulb. The lower mass $m_{2}$ is the sum of

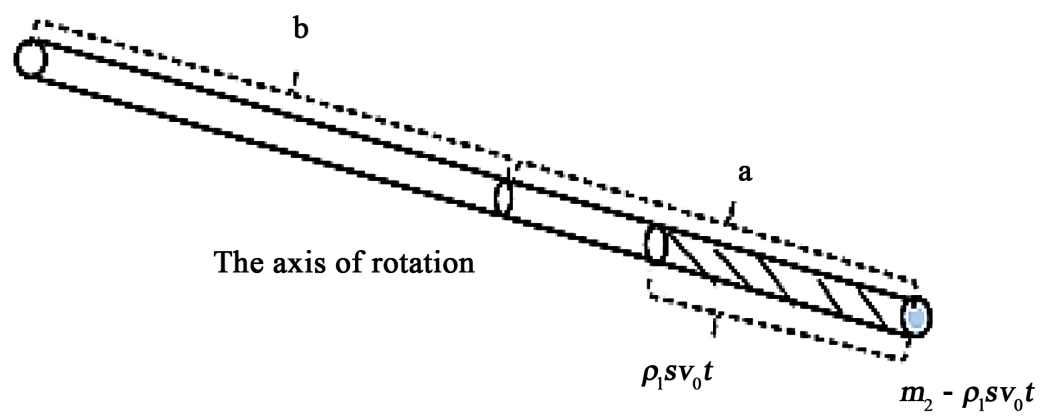

Figure 3. The time-dependent moment of inertia with upward motion of liquid. $\rho_{l}$ is density of $\mathrm{CH}_{2} \mathrm{Cl}_{2}, s$ is the cross-section of glass tube and $v_{0}$ is the upward velocity of liquid. 
masses of a glass bulb and liquid, and the density of liquid is specifically given by $\rho_{l} \sim 1.336 \mathrm{~g} / \mathrm{cm}^{3}$, and the cross-section of the inner glass tube is, $s=0.1256 \mathrm{~cm}^{2}$ (the internal radius $\sim 0.20 \mathrm{~cm}$ ) [3].

The mass of liquid is $\rho_{l} S v_{0} t$ and the mass at the lower bulb becomes $m_{2}-\rho_{l} s v_{0} t$. The moment of inertia for volatile water around the axis of rotation is given by the sum of moments from $a-v_{0} t$ to $a$ and $a^{2}\left(m_{2}-\rho_{l} s v_{0} t\right)$ given by the remaining mass at $a$ :

$$
\begin{aligned}
I_{2}(t) & =\int_{a-v_{0} t}^{a} r^{2} \mathrm{~d} m+a^{2}\left(m_{2}-\rho_{l} s v_{0} t\right) \quad\left(\mathrm{d} m=\rho_{l} s \mathrm{~d} r\right) \\
& =\rho_{l} s\left(\frac{a^{3}}{3}-\frac{\left(a-v_{0} t\right)^{3}}{3}\right)+a^{2}\left(m_{2}-\rho_{l} s v_{0} t\right) \\
& =a^{2} m_{2}\left(1-\frac{\rho_{l} s v_{0}^{2} t^{2}}{a m_{2}}+\frac{\rho_{l} s v_{0}^{3} t^{3}}{3 a^{2} m_{2}}\right) .
\end{aligned}
$$

Because of time-dependent volatile water distribution, the center of mass of $m_{2}$ is shifted from $a$ to $x_{2}(t)$ with time as:

$$
\begin{aligned}
x_{2}(t) & =\frac{1}{m_{2}}\left(\int_{a-v_{0} t}^{a} r \mathrm{~d} m+a\left(m_{2}-\rho_{l} s v_{0} t\right)\right) \quad\left(\mathrm{d} m=\rho_{l} s \mathrm{~d} r\right) \\
& =a-\frac{\rho_{l} s\left(v_{0} t\right)^{2}}{2 m_{2}}=a\left(1-\frac{\rho_{l} s v_{0}^{2} t^{2}}{2 a m_{2}}\right) .
\end{aligned}
$$

One should note that the shift, $x_{2}(t)$, should be substituted for $a$ in Equation (2.4), because $a$ in Equation (2.4) means the position of center of mass $m_{2}$ at $t=0$, corresponding to $x_{2}(0)=a$.

When liquid in the glass tube reaches the head, the mass of head, $m_{3}$, changes with time. It is denoted by $m_{3}^{*}(t)$ as:

$$
m_{3}^{*}(t)= \begin{cases}m_{3}+\rho_{l} s\left(v_{0} t-l\right) & \text { when } v_{0} t \geq l \\ m_{3} & \text { when } v_{0} t<l\end{cases}
$$

resulting in the moment of inertia of the head:

$$
I_{3}(t)=m_{3}^{*}(t) b^{2} .
$$

Now, the time-dependent moment of inertia is expressed as:

$$
I(t)=I_{0}+I_{2}(t)+I_{3}(t),
$$

and the substitution of $x_{2}(t)$ and $m_{3}^{*}(t)$ for $a$ and $m_{3}$ in (2.4) will yield:

$$
m^{*}(t)=m_{2} \frac{x_{2}(t)}{l}-m_{1}\left(\frac{b}{l}-\frac{1}{2}\right)-m_{3}^{*}(t) \frac{b}{l} .
$$

The numerical simulations are ready to be performed whether it is possible to produce periodic DB motions by adjusting parameters, $m_{1}, m_{2}, m_{3}, v_{0}, a, l$, and initial starting values are $\theta(0)=\pi / 6$ and $\dot{\theta}(0)=0$.

\subsection{Numerical Simulations for a Thermomechanical DB Motion}

By employing the result above, the equation of motion for the thermomechanical DB could be written as: 


$$
\ddot{\theta}+c \dot{\theta}+\frac{g l m^{*}(t)}{I(t)} \sin \theta=0,
$$

and the nonlinear differential Equation (3.7) should be compared with the mechanical equation of motion of (2.5). The Equations (2.5) and (3.7) are almost identical, except for time-dependent mass, $m^{*}(t)$ and moment of inertia, $I(t)$.

However, one must be careful that although the Equation (2.5) can be derived from Lagrngian (2.1), the Equation (3.7) cannot be derived from Lagrngian (2.1) with $m^{*}(t)$ and $I(t)$. A Lagrangian corresponding to (3.7) does not exist, and even if one could formally write down a complicated Lagrangian to obtain (3.7), it breaks the law of energy conservation fundamental for Newtonian mechanics. The energy of DB's motion is not conserved, or in other words, irreversibly dissipated, because the motion of a DB is an irreversible thermodynamic phenomena. This is the reason why we call the Equation (3.7) as a "thermomechanical model".

The thermomechanical model can produce DB's one periodic motion reasonably well, and the thermomechanical DB Equation (3.7) has a continuous solution changing from a standard periodic motion (oscillations around $\theta=0$ ) to a drinking motion which is converging to the upside down oscillations (oscillations around $\theta=\pi$ ) shown in Figure 4(a). However, in a real toy, the angle of bird's water dipping is restricted with the artificial shape of arm and axis of rotation in order to avoid upside-down solutions, and in addition, the angle resets the volatile water in the glass tube for next periodic motions. Hence, it is necessary to realize the same mechanism in numerical simulations.

When the angle of bird's water dipping $(\theta \simeq \pi / 2)$ resets volatile liquid in the glass tube, some mechanical properties of a DB are initialized (more or less initialized, because mechanical and thermodynamic state are not the same at all). The initialization in the equation of motion means that time-dependent
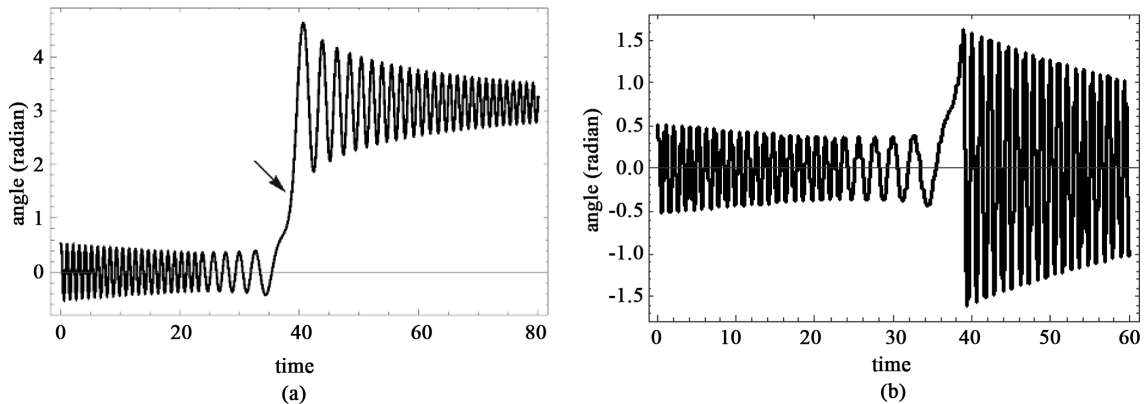

Figure 4. The one-periodic solution to a thermomechanical drinking bird defined by Equation (3.7), and (b) is the motion after initialization at $\theta \simeq \pi / 2$. (a) [The thermomechanical DB solution changes continuously from oscillations around $\theta=0$ to those around $\theta=\pi$ (upside-down oscillations). Initial starting values: $\theta(0)=\pi / 6$, $\dot{\theta}(0)=0$. The arrow indicates the initialization point $(t \simeq 38.8, \theta \simeq \pi / 2)$ for (b).] (b) [The initialization of bird's motion at $t \simeq 38.8$ seconds and $\theta \simeq \pi / 2$ in (a), resulting in oscillations after drinking.] 
quantities become

$$
\left(x_{2}(t), m^{*}(t), I(t)\right) \rightarrow\left(a, m^{*}, I_{0}\right)_{t_{1}},
$$

at a drinking time $t_{1}$. In order to initialize as (3.8), we use a step-function which is denoted as Unitstep $\left(t_{1}-t\right)$ :

$$
\text { Unitstep }\left(t_{1}-t\right)= \begin{cases}1 & t_{1} \geq t \\ 0 & t_{1}<t\end{cases}
$$

The time of initialization or drinking period, $t_{1}$, is determined from the solution to produce the bird's drinking angle $\theta \simeq \pi / 2$ in numerical simulations. By inserting the step-function in the drinking bird calculation, the initialization, $\left(x_{2}(t), m^{*}(t), I(t)\right) \operatorname{Unitstep}\left(t_{1}-t\right) \rightarrow\left(a, m^{*}, I_{0}\right)_{t_{1}}$, must be performed in the numerical calculation. The time $t_{1}$ indicates a period of bird's drinking time, and the step-function technique is also used for $m_{3}^{*}(t)$ in Equation (3.3), when volatile water in the glass tube reaches the bird's head.

A continuous transition of motion from a normal oscillation to an upside down oscillation is shown in Figure 4(a), and Figure $4(\mathrm{~b})$ is the one-periodic drinking motion initialized at time $t_{1} \simeq 38.8$ with the angle $\theta \simeq \pi / 2$. The oscillation becomes slow in the time range $25 \lesssim t \lesssim 40$ in Figure 4 (a), which is the time for the volatile water in the glass tube reaching the head and slowly increasing the moment of inertia for the bird head, and it corresponds to a slow periodic motion of a real toy before the bird's dipping water. When DB's water dipping and initialization are introduced at $t_{1}=38.8$, one obtains the Figure 4(b).

The period of drinking water is $T=38.8$ (second) and the number of oscillation (frequency) is $f=27$ in Figure 4. The periods of motion gradually change in time and elongate until the bird drinks water, and hence, the exact relations of period, frequency, wave length and velocity of rotation well-known in linear wave mechanics, are not obtainable. These values are sensitive to changes of parameter values and the velocity, $v_{0}$, of volatile water in the glass tube. The nonlinear equation of motion (3.7) produces forward $(\theta=\pi)$ and backward ( $\theta=-\pi$ ) upside down solutions depending on parameter values, and the normal oscillation around ( $\theta=0$ ) can be connected to those solutions by changing the value of artificial initialization time, $t_{1}$. For numerical calculations, it is better to make a check-list of how period, frequency, wave length are characteristically changed by the variations of parameters in Table 1.

Table 1. The parameter values for DB's one periodic motion in Figure 4(a). The parameters are listed in cgs-unit $(\mathrm{cm}, \mathrm{g}$, second). The gravitational constant, $\left(g=980 \mathrm{~cm} / \mathrm{s}^{2}\right)$ and constant of friction term $\left(c=0.4 \mathrm{~s}^{-1}\right)$ are fixed. The parameters to be adjusted are, masses: $m_{2}, m_{3},\left(m_{1}+m_{2}+m_{3}=28.0 \mathrm{~g}\right)$; velocity of liquid $v_{0}$ in the glass tube and lengths, $l$ and $a$. The ratio of masses $m_{3} / m_{2}=0.5$ is better for optimal performance of DB's motion. The Mathematica is used for numerical simulations.

\begin{tabular}{cccccc}
\hline$m_{1}(\mathrm{~g})$ & $m_{2}(\mathrm{~g})$ & $m_{3} / m_{2}$ & $v_{0}(\mathrm{~cm} / \mathrm{s})$ & $l(\mathrm{~cm})$ & $a(\mathrm{~cm})$ \\
\hline 7.0 & 14.0 & 0.5 & 0.25 & 12.4 & 6.0 \\
\hline
\end{tabular}




\subsection{Numerical Simulations for the Periodic Thermomechanical DB Motion}

The motion of a real DB toy is not accurately periodic like a spring or a pendulum, because initial and starting conditions for each DB's periodic oscillation are not completely the same; for example, the quantity of volatile water returned in the lower bulb, the effect of impacts of bird's beak at the edge of glass of water, temperature and humidity of environment are not identical at all. These varied conditions change the speed of volatile water in the glass tube, $v_{0}$, essential for the periodic oscillation, and hence, though all the starting conditions for the real DB's periodic motions are a little different, sufficiently stable periodic motions are produced.

The periodic thermomechanical DB motions (up to DB's 3-dipping motions) will be shown in the section, and they are sensitive to changes of parameter values and initialization timings, and one often obtains unexpected upside-down, two- or three-rotation solutions, which makes hard to control to obtain expected periodic solutions; one would need some time to get used to changing parameter values to obtain solutions shown in Figure 5. After one adjusted parameter values of initialization at $t_{1}$, conditions of initialization for the second and third period, $t_{2}, t_{3}$, are not simple $t_{2}=2 t_{1}, t_{3}=3 t_{1}$, but it is helpful to search the initialization time or dipping time. In Figure 5, the second and third periodic solutions are shown $\left(t_{1}=38.0, t_{2}=68.3\right.$ and $\left.t_{3}=101.3\right)$. Though the speed and amount of volatile water returned after bird's dipping are different in real DB motions, the velocity, $v_{0}$, of volatile water in the glass tube of thermomechanical DB is assumed constant, and the amount of volatile water returned from bird's dipping should also be adjusted in the model calculation.

In theoretical terms, the DB's motion is a dissipative and irreversible process, and mechanics and thermodynamics are integrated in the motion. Therefore, the drinking bird is not only fascinating but also scientifically fundamental in order to study fundamental principles. The thermomechanical model (3.7) could produce a useful mathematical expression to imitate DB's periodic motions, and properties of nonlinear equations with time-dependent coefficients are revealed.
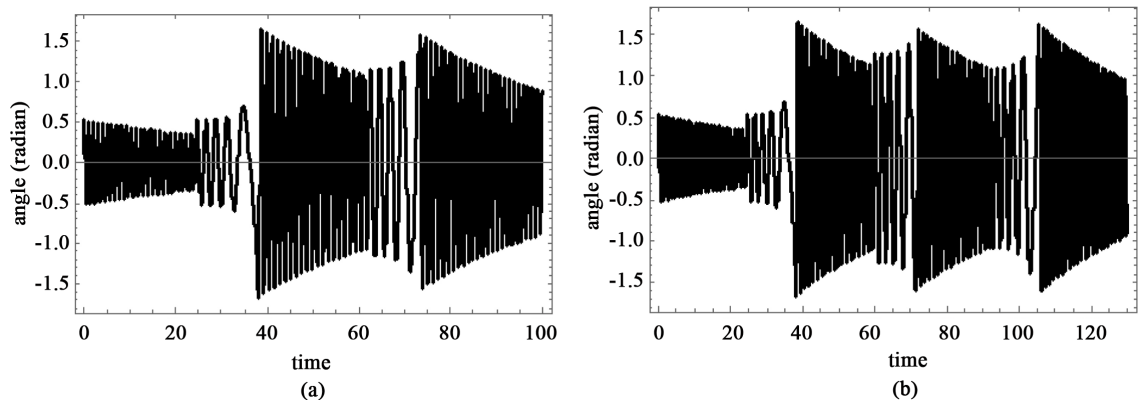

Figure 5. The DB solutions produced by the thermomechanical model (3.7). (a) [The thermomechanical DB solution with initialization at $t_{1}=38.0$ and $t_{2}=71.5$.] (b) [The thermomechanical DB solution with initialization at $t_{1}=38.0, t_{2}=68.3$ and $t_{3}=101.3$ ]. 
Other mathematical models could be constructed [3] for DB's motion. We have at least another model different from (3.7), and readers also could discover other different mathematical models for DB's periodic motions.

Although mathematical properties and structure of solutions are interesting, mathematics is only useful in science as a method of taxonomy in order to describe observations rigorously. The equation of thermomechanical model imitates DB's periodic motion reasonably well, and it would be useful for investigations of mechanism and related variables for understanding fundamental principles such as energy and entropy and possible electromechanical applications.

Based on the thermomechanical DB simulations, we would like to remark as:

(Remark 2)

The construction and duplication of the thermomechanical drinking bird show essentially complementary relations between Newtonian mechanics and thermodynamics to operate natural motions by way of energy, speed of matter, heat and temperature. Whatever principles we could conjecture to explain thermomechanical phenomena, natural phenomena are more fundamental than any consistency of humane logical thought-experiment.

\section{Conclusions and Perspectives}

The more we study DB's motions, the more we realize that it is scientifically interesting and enlightening in terms of physical principles and applications. It would help people in science reflect on physical principles and science, such as energy and entropy, status of mathematics in science, fundamental meanings of applicability and application. The drinking bird also encouraged us to consider fundamental logical structure of science, regarding reproducibility, self-consistency and testability. Reproducibility means exact recordings of experiments (mathematics as taxonomy), correct reproductions of observed data and natural phenomena. Self-consistency means no contradictions within a model as theoretical consistency and calculations. Testability includes experimental and possible theoretical tests as well as specific applications in science; applications are required for testability, which must not be taken for granted. These three criteria are essential to define science.

Although mathematical analyses combined with supercomputers and experimental technologies are helpful and powerful instruments to observe natural phenomena, they correspond to taxonomy in science: exact reproductions, expressions and calculations. One should always have in mind that physical principles and laws to maintain three criteria are fundamental and ultimate entity for science.

It should be emphasized that mathematical properties (nonlinearity, differential equations, ...) and supercomputer simulations of natural phenomena are only for the purpose of taxonomy and correct recordings of observations. Beyond taxonomy, we have to test our models to ultimately understand dynamics and principles of nature, which is the most fundamental property in science. The 
DB's periodic motion can be mathematically expressed, however, it is not the end of the problem, the beginning of pursuit of fundamental physics. The end of science or physics is in mind of people who believe that the end of physics exists. Natural phenomena are beyond human imagination.

The theory of DB's irreversible thermodynamics is fundamental and applicable to any structure considered to be a heat engine from microscopic to macroscopic scales, such as the energy flow of nuclear energy reactors, flow of electric current through semiconductors, thermocouples, chemical reactions and electron transport processes in batteries, energy conversions of biological systems, etc. Hence, it is interesting to conjecture thermomechanical principles, laws and relations to quantum electrodynamics (QED) and quantum hadrodynamics (QHD) [17] [18]. The fundamental physics is not only in high energy phenomena, but also in DB's motion, ordinary and routine natural science.

It is fundamental to check the theory of irreversible thermodynamics, applications and applicability manifested in the DB analysis, and it could be a possible theoretical foundation for new applications to renewable and sustainable energy harvesting technologies [19]. Similarly, applications of a Stirling engine to sustainable energy technologies as thermodynamic theory of heat engine can be investigated. Temperature and pressure of a container sealed with a working fluid are measured by remote sensor measurement. It is possible to establish a power generation system by providing a large temperature and pressure difference in the container [20].

A drinking bird is a fascinating toy to understand and appreciate possibility of the theory of irreversible thermodynamics, more fascinating than we discussed in the paper. It encourages us to study fundamental physics, mathematics and logic in science, and we realize that this is an appropriate concluding remark in this study.

\section{Acknowledgements}

We would like to acknowledge Dr. J. Denur at Department of Physics, University of North Texas, USA, for his valuable comments on the article.

\section{Conflicts of Interest}

The authors declare no conflicts of interest regarding the publication of this paper.

\section{References}

[1] Bucky, P.A. (1992) The Private Albert Einstein. Andrews and McMeel, Kansas City, MO.

[2] Murrow, R.M. (1966) A Simple Heat Engine of Possible Utility in Primitive Environment. The Rand Corporation, Santa Monica, CA.

[3] Güémeza, J., Valiente, R., Fiolhais, C. and Fiolhais, M. (2003) Experiments with the Drinking Bird. American Journal of Physics, 71, 1257.

https://doi.org/10.1119/1.1603272 
[4] Lorenz, R. (2006) Finite-Time Thermodynamics of an Instrumented Drinking Bird Toy. American Journal of Physics, 74, 677. https://doi.org/10.1119/1.2190688

[5] Førland, K.S., Førland, T. and Ratkje, S.K. (1988) Irreversible Thermodynamics; Theory and Applications. John Wiley \& Sons, New York.

[6] Lovering, D.G. (1982) Molten Salt Technology. Springer Science + Business Media, New York. https://doi.org/10.1007/978-1-4757-1724-2

[7] Gaune-Escard, M. and Haarberg, G.M. (2014) Molten Salts Chemistry and Technology, John Wiley \& Sons, New York. https://doi.org/10.1002/9781118448847

[8] Rubin, A. and Riznichenko, G. (2014) Mathematical Biophysics. Springer, Dordrecht. https://doi.org/10.1007/978-1-4614-8702-9

[9] Uechi, L. and Uechi, H. (2016) Noether's Conservation Laws and Stability in Nonlinear Conservative Interactions. Open Access Library Journal, 3, e2592.

[10] Sieniutycz, S. (1994) Conservation Laws in Variational Thermo-Hydrodynamics. Kluwer Academic Publishers, Dordrecht, The Netherlands.

[11] Poincaré, H. (2019) Bifurcation Theory. (Wikipedia) https://en.wikipedia.org/wiki/Bifurcationtheory

[12] Peters, A.D., Jaffe, C. and Delos, J.B. (1994) Quantum Manifestations of Bifurcations of Classical Orbits: An Exactly Solvable Model. Physical Review Letters, 73, 2825. https://doi.org/10.1103/PhysRevLett.73.2825

[13] Gao, J. and Delos, J.B. (1997) Quantum Manifestations of Bifurcations of Closed Orbits in the Photoabsorption Spectra of Atoms in Electric Fields. Physical Review $A, 56,356$. https://doi.org/10.1103/PhysRevA.56.356

[14] Wells, D.A. (1967) Lagrangian Dynamics. McGraw-Hill, New York.

[15] Goldstein, H. (1980) Classical Mechanics. Addison-Wesley, Boston, MA.

[16] Video, Wikipedia (2019) The Engineering of the Drinking Bird. The Mechanical Structure and Motion of a Drinking Bird Is Experimentally Well Exposed. https://en.wikipedia.org/wiki/Drinking bird

[17] Serot, B.D. and Walecka, J.D. (1986) Advances in Nuclear Physics. Volume 16, Plenum, New York.

[18] Uechi, S.T. and Uechi, H. (2017) Self-Consistent Many-Body Theory and Nuclear Matter in a Chiral Dirac-Hartree-Fock Approximation. Quarterly Physics Review, 3, 1-19.

[19] Uechi, S.T. and Uechi, H. (2019) A Mechanical Vibration-Induced Electric Energy Generation (MVEG) and Applications to Ride Quality of Vehicles and International Roughness Index (IRI). Studies in Engineering and Technology, 6, 59-69. https://doi.org/10.11114/set.v6i1.4301

[20] Nishimura, A., et al. (2017) Thermal Management of Heat Resistant FBG Sensing for High Temperature Industrial Plants? E-Journal of Advanced Maintenance, 9, 52-59. http://www.jsm.or.jp/ejam/Vol.9No.2/AA/AA128/AA128.html 